\title{
La participación de las familias en educación infantil
}

Family involvement in early childhood education

\author{
Parra, Joaquín; Gomariz, María-Ángeles; Hernández-Prados, María-Ángeles \& García-Sanz, \\ Mari-Paz \\ Universidad de Murcia (España)
}

\begin{abstract}
Resumen
Los beneficios que aporta la implicación familiar en el proceso educativo de los hijos y su repercusión en la calidad de la educación son hechos ampliamente demostrados. El objetivo de este estudio fue conocer posibles agrupamientos de familias, cuyos hijos cursan la etapa de Educación Infantil, configurados a partir de una serie de variables de participación familiar y sociodemográficas. Para ello, se contó con 1.953 familias españolas, a las que se les aplicó un cuestionario ad hoc de 66 ítems, con alta fiabilidad. Tras realizar un análisis clúster, los resultados ofrecieron cuatro perfiles familiares: 1) perfil participativo no normativo, 2) perfil no participativo de nacionalidad no española, 3) perfil participativo normativo y 4) perfil no normativo de nacionalidad española. El perfil más difuso es el 2, no hallándose diferencias significativas del resto con este perfil. Asimismo, la magnitud de las diferencias entre algunos perfiles no queda totalmente definida.
\end{abstract}

Fecha de recepción 2016 Noviembre 19

Fecha de aprobación 2017 Junio 8

Fecha de publicación 2017 Junio 9

\section{Palabras clave:}

Familia, escuela, Educación Infantil, participación.

\begin{abstract}
The benefits of the family implication in the educational process of children and its repercussion in the quality of the education are facts widely demonstrated. The aim of this research knew the range of possible groups whose children study in the stage of childhood education, formed from a set of variables of family participation and sociodemographics. Thus, this research counted on the help of 1953 Spanish families by means of a questionnaire ad hoc of 66 items, with high reliability. After further Cluster Analysis, the results show four family profile: 1) participative profile non normative, 2) non participative profile of not Spanish nationality, 3) participative profile normative 4) non normative profile of Spanish nationality. The more diffuse profile is number two, with no significant differences with the rest of the profiles. In addition, the magnitude of the differences between some profiles is not totally defined.
\end{abstract}

Reception Date 2016 November 19

Approval Date 2017 June 8

Publication Date: 2017 June 9

\section{Keywords:}

Family, school, kindergarten, participation.

Son múltiples los antecedentes en los que se reconoce la participación de las familias en los centros escolares como uno de los indicadores de calidad educativa. Así, en ocasiones se ha entendido que una escuela eficaz es 'aquella que consigue un desarrollo integral de todos y cada uno de sus estudiantes, más allá de lo que sería previsible teniendo en cuenta su rendimiento previo y la situación socio- económica y cultural de sus familias’ (Murillo, 2005, 30). Como se aprecia, el propio concepto de escuela eficaz hace alusión al papel educativo de la familia.

Existen evidencias de que la alta implicación positiva de las familias del alumnado en las escuelas está relacionada con una mayor eficacia de los centros escolares, de ahí el esfuerzo en promocionar dicha 
participación para que se asiente como rasgo identificativo del centro, ya que se considera una buena práctica. Pero aún queda por conocer el carácter de esta implicación, cómo se establece y si existe más de un factor que interfiera de forma causal (Azpillaga, Intxausti \& Joaristi, 2014).

Desafortunadamente, la participación de las familias no se mantiene constante a lo largo de toda la escolaridad. Tal y como señala Peñalver (2009), esta participación adquiere un mayor protagonismo en Educación Infantil, donde la implicación de las familias suele ser más intensa que en Primaria y Secundaria (Garreta, 2008; Gomariz, Parra, García, Hernández \& Pérez, 2008), produciéndose un seguimiento más directo y una relación más cordial y cercana con el profesorado (Panigua, 2009).

Sin lugar a dudas son muchos los aspectos que favorecen esta implicación inicial de los padres, e igualmente numerosos los factores que impiden el mantenimiento de la misma en etapas posteriores. Desearíamos señalar, en primer lugar, que los propios beneficios que se asocian a la participación de las familias en las cuestiones educativas de los hijos actúan como catalizador de dicha participación. En este sentido, la participación familiar facilita el proceso de aprendizaje, mejorando el rendimiento académico y contribuyendo a reducir las cifras de fracaso escolar (Sheldon \& Epstein, 2002).

En segundo lugar, reconocer que existe en nuestra sociedad una disposición ético-moral a atender la vulnerabilidad de los niños, algo que no ha existido siempre, pero que desde la Declaración Universal de los Derechos del Niño (1959) concurre una gran sensibilización al respecto. De ahí que culturalmente exista la tendencia de que los padres han de atender y proteger a sus hijos proporcionando las condiciones idóneas para su desarrollo y potenciación como ser humano, considerando que se trata de una cuestión de responsabilidad educativa ineludible.

Conforme los niños avanzan en edad y escolaridad, se produce una merma del acompañamiento e implicación familiar, bajo la pretensión y finalidad educativa de promover el desarrollo de la autonomía. Ambos conceptos se han entendido en nuestra cultura como antagónicos, cuando en realidad pueden y deben complementarse. Todos estamos necesitados de apoyo, de acompañamiento. Se trata de un sentimiento natural y una necesidad inherente a la propia condición inacabada del ser humano. "El individuo no vive en el vacío. Vive en comunidad. Pero la comunidad es el conjunto de individuos que interactúan voluntariamente” (Gaviria, 2014:14). Así, la educación no debe entenderse independiente de los contextos y agentes implicados, sino como una coeducación en la que voluntariamente tienen cabida todos ellos. Desde este posicionamiento, se trata de promover redes de comunicación y cooperación entre familia y escuela, asentadas sobre la confianza y la libertad, y motivados por el objetivo común de integrar a las nuevas generaciones en la sociedad.

La satisfacción de esta mayor participación en la etapa de Educación Infantil, no puede obviar la necesidad de continuar mejorando esta dimensión. La corresponsabilidad de la educación requiere crear comunidades de objetivos y prácticas compartidas (Paniagua, 2009), ampliar los canales de comunicación y colaboración activa entre centro, docentes y familias, aumentar el sentido de pertenencia, promover la implicación directa e indirecta de las familias en los aprendizajes... Requiere, pues, de la sensibilización y formación necesaria para enseñar a participar, tanto a docentes como a familias, y ser capaces de transmitir ese valor. Las nuevas generaciones deben tener ejemplos de participación de sus contextos cercanos, si queremos que la ciudadanía del futuro sea comprometida y participativa.

Con la intención de conocer cuáles son los tipos, las formas o los perfiles de participación familiar en Educación Infantil y las variables que caracterizan a estas familias, nos adentramos en este estudio. Así pues, se han 
contemplado variables referidas a una participación no normativa, como son la comunicación y participación tanto en el centro educativo como desde el hogar, incorporando elementos valorativos de frecuencia y satisfacción que en definitiva nos informan de su calidad; variables centradas en las actitudes positivas, el sentido de pertenencia y el compromiso de las familias con la comunidad (Fullan \& Stiegielbauer, 2003); y por último, variables relacionadas con las implicaciones indirectas de las familias a través de las asociaciones o los órganos de consulta o decisión en los centros, a la que podemos denominar participación normativa.

Por otra parte, en lo que respecta a las variables situacionales o sociodemográficas concretas que nos permiten dibujar los perfiles familiares, nos hemos cuestionado, junto con otros autores, aspectos referidos al nivel socioeconómico y cultural de las familias, así como el nivel de estudios de los padres (Bolívar, 2006; Pérez-Díaz, Rodríguez \& Sánchez, 2001) en los que se ha reflexionado sobre el papel y la importancia que desempeña el capital humano, recordando el informe Coleman de los ochenta. Otras variables consideradas han sido la edad de los progenitores, la titularidad de los centros a los que acuden (Pérez-Díaz et al., 2001) o el origen nacional de las familias (Rodríguez, 2010a; Rodríguez, 2010b; Santos \& Lorenzo, 2009), con el deseo de que se haya conseguido 'romper' con esas dinámicas sociales que se retroalimentan y que dificultan confluir en la inclusión (Escudero, 2006; Instituto de Formación del Profesorado, Investigación e Innovación Educativa, 2011).

Teniendo en cuenta todo lo anterior, el presente trabajo se ubica en una investigación más amplia coordinada por el Consejo Escolar del Estado (2014), en la que participaron cerca de 15.000 familias, cuyos hijos e hijas se encuentran escolarizados en las etapas educativas de Educación Infantil, Primaria y Secundaria. El propósito concreto del estudio que se muestra en este artículo fue conocer los perfiles de participación en la educación escolar que caracteriza a grupos de familias del alumnado que cursa la etapa de Educación Infantil. En función de sus rasgos más sobresalientes y utilizando los análisis estadísticos oportunos, la hipótesis de este estudio ha querido demostrar la existencia de agrupamientos de familias que giran en torno a variables como la titularidad del centro o el origen nacional de sus miembros, sin perder de vista ese capital humano y social al que nos hemos referido en párrafos anteriores.

\section{Método}

\section{Participantes}

La investigación más amplia a la que nos hemos referido se desarrolló considerando el conjunto del Estado Español mediante un muestreo aleatorio estratificado a partir de las variables: 'etapa educativa' y 'titularidad del centro’. Centrándonos únicamente en la etapa de Educación Infantil, en este estudio participaron 2.452 familias de 93 centros educativos, de las cuales fueron excluidas 508 por no generar datos válidos. Así pues, la muestra real fue de 1.944 familias.

En la Tabla 1 se indica, porcentualmente, la distribución muestral de los padres y madres participantes, en función de las variables sociodemográficas consideradas en el estudio, cuyos datos serán comentados en el apartado de Resultados de este artículo, una vez explicitado el significado de las variables indicadas en la misma. 
Tabla 1. Distribución muestral de las familias de Educación Infantil.

\begin{tabular}{|c|c|c|}
\hline Variables & Categorías & $\begin{array}{l}\text { Porcentaje de } \\
\text { familias }\end{array}$ \\
\hline \multirow{3}{*}{ Titularidad del centro } & Pública (1) & $63,2 \%$ \\
\hline & Concertada (2) & $34,6 \%$ \\
\hline & Privada (3) & $2,2 \%$ \\
\hline \multirow{2}{*}{ Nacionalidad de la familia } & España (1) & $81,1 \%$ \\
\hline & Otro país (2) & $17,5 \%$ \\
\hline \multirow{5}{*}{ Edad del padre } & Menos de 20 años (1) & $0 \%$ \\
\hline & De 20 a 30 años (2) & $5,3 \%$ \\
\hline & De 31 a 40 años (3) & $58,7 \%$ \\
\hline & De 41 a 50 años (4) & $30,3 \%$ \\
\hline & Más de 51 años (5) & $1,4 \%$ \\
\hline \multirow{5}{*}{ Edad de la madre } & Menos de 20 años (1) & $0,1 \% \%$ \\
\hline & De 20 a 30 años (2) & $11,8 \%$ \\
\hline & De 31 a 40 años (3) & $70,1 \%$ \\
\hline & De 41 a 50 años (4) & $15,9 \%$ \\
\hline & Más de 51 años (5) & $0 \%$ \\
\hline \multirow{8}{*}{ Estudios del padre } & Primaria sin completar (1) & $3,3 \%$ \\
\hline & Primaria (2) & $12,7 \%$ \\
\hline & Secundaria obligatoria (3) & $16,2 \%$ \\
\hline & Bachillerato o FP grado medio (4) & $20,7 \%$ \\
\hline & FP grado superior (5) & $13,9 \%$ \\
\hline & Diplomatura (6) & $7,5 \%$ \\
\hline & Licenciatura o similar (7) & $15,7 \%$ \\
\hline & Doctorado (8) & $1,4 \%$ \\
\hline \multirow{8}{*}{ Estudios de la madre } & Primaria sin completar (1) & $2,3 \%$ \\
\hline & Primaria $(2)$ & $8,6 \%$ \\
\hline & Secundaria obligatoria (3) & $12,7 \%$ \\
\hline & Bachillerato o FP grado medio (4) & $20,8 \%$ \\
\hline & FP grado superior (5) & $13,9 \%$ \\
\hline & Diplomatura (6) & $16,5 \%$ \\
\hline & Licenciatura o similar (7) & $19 \%$ \\
\hline & Doctorado (8) & $1,3 \%$ \\
\hline \multirow{6}{*}{$\begin{array}{l}\text { Número de libros } \\
\text { en casa }\end{array}$} & Entre 0 y 10 libros (1) & $7,9 \%$ \\
\hline & Entre 11 y 25 libros (2) & $15,9 \%$ \\
\hline & Entre 26 y 100 libros (3) & $39,9 \%$ \\
\hline & Entre 101 y 200 libros (4) & $17,2 \%$ \\
\hline & Entre 201 y 500 libros (5) & $11,6 \%$ \\
\hline & Más de 500 libros (6) & $4,3 \%$ \\
\hline \multirow{4}{*}{ Recursos de aprendizaje } & Menos de 3 recursos (1) & $4,2 \%$ \\
\hline & De 3 a 6 recursos (2) & $27,2 \%$ \\
\hline & De 7 a 10 recursos (3) & $47,4 \%$ \\
\hline & Más de 10 recursos (4) & $21,2 \%$ \\
\hline \multirow{4}{*}{ Otros recursos materiales } & Menos de 10 puntos (1) & $26,7 \%$ \\
\hline & De 10 a 20 puntos (2) & $68,8 \%$ \\
\hline & De 21 a 30 puntos (3) & $1,5 \%$ \\
\hline & Más de 30 puntos (4) & $0,1 \%$ \\
\hline
\end{tabular}




\section{Instrumento}

Se aplicó un cuestionario, en formato papel en los propios centros y remitidos al Consejo Escolar del Estado por correo postal, como impulsor y gestor administrativo del estudio. El cuestionario completo consta de 141 ítems de distintos tipos, si bien para esta investigación se utilizaron 66 ítems, 45 relativos a la participación familiar en el proceso educativo de los hijos e hijas y 21 referidos a datos sociodemográficos.

La validez de contenido del cuestionario quedó salvaguardada por la participación de un grupo de expertos de cuatro universidades españolas, quienes participaron en la validación a través de juicio de expertos del instrumento, atendiendo fundamentalmente a su claridad y relevancia.

La fiabilidad del instrumento completo para una muestra de 14.876 familias, en las que se incluyeron niveles de Educación Primaria y Secundaria Obligatoria, junto con el de Educación Infantil, arrojó un coeficiente alpha de Cronbach de 0,867 , lo que indica una consistencia interna alta del cuestionario (De Vellis, 2003). Para la muestra de familias cuyos hijos e hijas cursaban Educación Infantil, la fiabilidad resultó ser prácticamente la misma (alpha de Cronbach $=0,864$ ).

\section{Variables}

Para acotar los perfiles de participación se contemplaron, en primer lugar, variables que hacen alusión directa a dicha participación que mantienen las familias con el centro educativo de sus hijos: calidad de la comunicación, participación en actividades del centro, sentimiento de pertenencia, implicación directa desde el hogar, implicación en la AMPA e implicación en el Consejo Escolar. El número de ítems y contenido de cada una de estas variables es el siguiente:

- 'Calidad de la comunicación': se consideraron 9 ítems (1: Nunca - 4: Siempre), que integran aspectos relacionados con la frecuencia con la que se comunican las familias con el centro, así como el nivel de satisfacción que las mismas encuentran con dicha comunicación (p.e. "asisto a las tutorías cuando me cita el tutor/a de mi hijo/a" o "considero satisfactorias las reuniones que mantengo con el centro”).

- 'Participación de las familias en las actividades concretas que organiza el centro': se contemplaron 4 ítems (1: Desconozco si el centro organiza - 4: Colaboro y participo) relativos a la participación familiar en actividades formativas para padres y madres, comisiones de trabajo creadas en el centro o actividades de apoyo al centro (p.e. "participo en actividades formativas para padres y madres como escuelas de padres y madres, charlas o conferencias divulgativas, etc.”).

- 'Sentimiento de pertenencia': esta variable quedó configurada por 4 ítems, mediante los cuales se preguntó a las familias (1: Desacuerdo absoluto - 4: Mayor acuerdo) si se sentían parte del centro, si lo consideraban como algo suyo (p.e. "si un equipo del centro participa en un deporte, un concurso..., ese es mi equipo”).

- 'Implicación desde el hogar': se consideraron 12 cuestiones (1: Nunca 4: Siempre) que hacen referencia a la colaboración y acompañamiento educativo que las familias realizan con sus hijos e hijas desde en sus casas (p.e. "comparto objetivos educativos comunes con el centro para desarrollarlos en casa" o "en mi familia vamos al cine, teatro, museos, viajes, conciertos, exposiciones, etc.”).

- 'Implicación a través de la AMPA': quedó constituida por 8 ítems (0: No - 1: Sí) que versan sobre el conocimiento que las familias tienen de dicha asociación, su participación en las distintas actividades que desarrolla 0 el 
compromiso adquirido con la misma (p.e. "soy o he sido socio de alguna AMPA en el centro en el que está escolarizado mi hijo/a”).

- 'Implicación en los Consejos Escolares', variable también integrada por 8 ítems (0: No - 1: Sí), mediante preguntas respecto al conocimiento que las familias tienen sobre ellos, las funciones que desempeñan o quiénes son sus miembros, su participación en las elecciones, etc. (p.e. "Conozco a los representantes de las familias en el Consejo Escolar del centro").

De estas 6 variables, las cuatro primeras se obtuvieron a través de la media de los ítems utilizados para su configuración, mientras que las dos últimas resultaron del sumatorio de las respuestas afirmativas obtenidas en los ítems que las conforman.

En segundo lugar, hemos considerado otras variables que recogen información sobre las situaciones sociodemográficas de las familias (ver Tabla 1), tales como: titularidad del centro (pública, concertada y privada), nacionalidad de las familias (española y no española), edad del padre (en intervalos con una escala de 1 a 5), edad de la madre (escala de 1 a 5), número de libros en casa (escala de 1 a 6), estudios del padre (escala de 1 a 8), estudios de la madre (escala de 1 a 8 ), recursos para el aprendizaje (escala de 1 a 4) y recursos materiales (escala de 1 a 5).

En el apartado de Participantes se ha indicado el número de opciones o categorías que las familias podían elegir para responder a este tipo de variables. Cada una de ellas se corresponde con un ítem del cuestionario, a excepción de las referidas a los recursos para el aprendizaje y a los recursos materiales.

La variable "recursos para el aprendizaje" (sin contar el número de libros) quedó configurada por el sumatorio de 12 ítems en los que se preguntaba por la disponibilidad en los hogares de elementos como: una habitación propia, un lugar tranquilo para estudiar, un ordenador para realizar las tareas escolares, software educativo, conexión a internet, libros de literatura clásica, de arte, etc. Aunque somos conscientes de que no todos estos recursos tienen el mismo valor (ni educativo, ni económico), no discriminamos ninguno frente al resto, ya que consideramos que ello podía ser demasiado subjetivo. Para la medición de esta variable se consideraron 4 intervalos (ver Tabla 1).

La variable "recursos materiales" (otros, además de los mencionados) se conformó a partir de 4 ítems: número de teléfonos móviles, de televisiones, de ordenadores y de coches. Esta variable también se obtuvo mediante el sumatorio de los 4 ítems que la integran, pero en esta ocasión, sí se otorgó un valor ponderado a cada recurso. En este sentido, siendo también conscientes de que dicha ponderación depende de marcas, tamaños y otras características de cada recurso, consideramos por igual los teléfonos móviles, las televisiones y los ordenadores, pero cada coche se contabilizó como 4 de los restantes recursos, ya que, lo más importante era conocer el nivel económico de las familias. Los resultados se contabilizaron como puntos, estableciéndose para la medición de esta variable 4 intervalos (ver Tabla 1).

\section{Procedimiento}

Si bien fue el Consejo Escolar del Estado quien encargó al equipo de investigación el desarrollo de un estudio general respecto a la familia y centros educativos, es el equipo quien concretó los objetivos, así como el diseño del cuestionario para la recogida de información. Una vez validado el instrumento, fue contratada una empresa para la distribución y posterior recogida de los cuestionarios en los centros seleccionados. Se procedió al vaciado de los cuestionarios y la generación de los archivos de datos en formato SPSS y se efectuaron los análisis pertinentes. 


\section{Análisis de datos}

Se realizó un análisis cluster en dos fases o bietápico. La elección de esta técnica dentro del análisis de conglomerados se debe a que el algoritmo que emplea la misma, coincidiendo con las características de nuestros datos, permite el tratamiento simultáneo de variables categóricas y cuantitativas, determinar automáticamente el número óptimo de conglomerados y analizar archivos de datos de gran tamaño (Pérez López, 2009). Los cálculos inferenciales se han realizado con un nivel de significación estadística de $\alpha=0,05$.
Para obtener el índice $d$ de Cohen (1988) se ha recurrido a la calculadora del tamaño del efecto EasyCalculation.com (s.f.).

\section{Resultados}

En función del propósito de la investigación, se obtuvieron cuatro conglomerados de participación de las familias del alumnado de Educación Infantil (1, 2, 3 y 4), en los que se adscriben unos determinados porcentajes de familias (Tabla 2).

Tabla 2. Distribución de conglomerados. Conglomerados de participación de las familias de Educación Infantil

\begin{tabular}{ccccc}
\hline & & $\mathrm{N}$ & \% de combinados & \% del total \\
\hline & 1 & 643 & $33,1 \%$ & $26,2 \%$ \\
\cline { 2 - 5 } Conglomerado & 2 & 304 & $15,6 \%$ & $12,4 \%$ \\
\cline { 2 - 5 } & 3 & 531 & $27,3 \%$ & $21,7 \%$ \\
\cline { 2 - 5 } & 4 & 466 & $24,0 \%$ & $19,0 \%$ \\
\hline \multirow{2}{*}{ Combinados } & 1.944 & $100,0 \%$ & $79,3 \%$ \\
\hline Total & 508 & & $20,7 \%$ \\
\hline
\end{tabular}

Seguidamente, en las tablas 3,4 y 5 se presentan las medias y desviaciones típicas o los porcentajes de las variables consideradas, en función de los cuatro conglomerados obtenidos. Fue a través de estas variables cómo se definieron las formas de participación de las familias y las características esenciales que nos ayudaron a definirlas. 
Tabla 3. Descriptivos de las variables numéricas por conglomerados en Educación Infantil. Medias y desviaciones típicas.

\begin{tabular}{|c|c|c|c|c|c|c|}
\hline \multirow{2}{*}{ Variable } & \multirow{2}{*}{ Estadístico } & \multicolumn{5}{|c|}{ Conglomerado } \\
\hline & & 1 & 2 & 3 & 4 & Combinados \\
\hline \multirow{2}{*}{$\begin{array}{l}\text { Calidad de la } \\
\text { comunicación }\end{array}$} & Media & 2,80 & 2,65 & 2,90 & 2,58 & 2,75 \\
\hline & Desv. típica & ,401 & ,447 & ,402 & ,322 & ,411 \\
\hline \multirow{2}{*}{$\begin{array}{l}\text { Participación } \\
\text { actividades }\end{array}$} & Media & 2,29 & 2,03 & 2,46 & 1,86 & 2,19 \\
\hline & Desv. típica & ,785 & ,759 & ,767 & ,647 & ,780 \\
\hline \multirow{2}{*}{$\begin{array}{l}\text { Sentimiento } \\
\text { de pertenencia }\end{array}$} & Media & 3,21 & 3,14 & 3,36 & 2,86 & 3,16 \\
\hline & Desv. típica & 611 & 660 & ,530 & ,647 & ,633 \\
\hline \multirow{2}{*}{$\begin{array}{l}\text { Colaboración } \\
\text { hogar }\end{array}$} & Media & 3,51 & 3,36 & 3,59 & 3,29 & 3,45 \\
\hline & Desv. típica & ,312 & ,407 & ,257 & ,332 & ,3407 \\
\hline \multirow{2}{*}{$\begin{array}{l}\text { Implicación } \\
\text { AMPA }\end{array}$} & Media & 2,19 & 1,93 & 3,73 & 1,62 & 2,43 \\
\hline & Desv. típica & 1,995 & 2,006 & 2,314 & 1,447 & 2,141 \\
\hline \multirow{2}{*}{$\begin{array}{l}\text { Implicación } \\
\text { CE }\end{array}$} & Media & 2,03 & 1,97 & 3,77 & 1,79 & 2,44 \\
\hline & Desv. típica & 1,933 & 2,060 & 2,344 & 1,632 & 2,170 \\
\hline \multirow{2}{*}{ Edad padre } & Media & 3,33 & 3,34 & 3,37 & 3,15 & 3,30 \\
\hline & Desv. típica & ,551 & ,690 & ,521 & ,578 &, 580 \\
\hline \multirow{2}{*}{ Edad madre } & Media & 3,14 & 2,98 & 3,17 & 12,88 & 3,06 \\
\hline & Desv. típica & ,493 & ,551 & ,440 & ,540 &, 514 \\
\hline \multirow{2}{*}{$\begin{array}{l}\text { Estudios } \\
\text { padre }\end{array}$} & Media & 4,79 & 4,15 & 4,80 & 3,45 & 4,37 \\
\hline & Desv. típica & 1,761 & 1,746 & 1,768 & 1,525 & 1,797 \\
\hline \multirow{2}{*}{$\begin{array}{l}\text { Estudios } \\
\text { madre }\end{array}$} & Media & 5,27 & 4,57 & 5,33 & 3,91 & 4,85 \\
\hline & Desv. típica & 1,606 & 1,726 & 1,537 & 1,594 & 1,708 \\
\hline \multirow{2}{*}{$\begin{array}{l}\text { Número de } \\
\text { libros }\end{array}$} & Media & 3,50 & 2,90 & 3,69 & 2,74 & 3,28 \\
\hline & Desv. típica & 1,169 & 1,282 & 1,123 & ,996 & 1,202 \\
\hline \multirow{2}{*}{$\begin{array}{l}\text { Recursos de } \\
\text { aprendizaje }\end{array}$} & Media & 3,06 & 2,84 & 3,23 & 2,55 & 2,95 \\
\hline & Desv. típica & ,706 & ,773 & ,645 & ,635 & ,731 \\
\hline \multirow{2}{*}{$\begin{array}{l}\text { Recursos } \\
\text { materiales }\end{array}$} & Media & 1,77 & 1,66 & 1,87 & 1,70 & 1,76 \\
\hline & Desv. típica & ,445 & ,544 & ,379 &, 516 & ,469 \\
\hline
\end{tabular}

En la Tabla 3, en la columna denominada de 'combinados' (que representa la totalidad de familias de Educación Infantil) se aprecia que las familias, globalmente, perciben bastante alta la calidad en la comunicación con el centro educativo ( $\bar{X}=2,75$, sobre 4$)$, participan poco en actividades organizadas por dicho centro $(\bar{X}=2,19$, sobre 4$)$, poseen un elevado sentimiento de pertenencia hacia el mismo ( $\bar{X}=3,15$, sobre 4 ), colaboran entre bastante y mucho de manera directa desde el hogar $(\bar{X}=3,45$, sobre 4$)$ y se implican moderadamente, tanto en la AMPA $(\bar{X}=2,43$, sobre 8) como en el Consejo Escolar del centro $(\bar{X}=2,44$, sobre 8$)$. 
En la misma columna de "combinados" de la Tabla 3, apoyada en la Tabla 1 (apartado de "Participantes"), se comprueba que se trata de familias donde los padres tienen una edad media aproximada de 43 años $(\bar{X}=3,30)$ y las madres sobre 40 años $(\bar{X}=3,06)$, con estudios medios del padre situados entre el nivel de Bachillerato o Formación Profesional (FP) de Grado Medio y FP de Grado Superior $(\bar{X}=$ 4,37) y de la madre en este último nivel $(\bar{X}=$
4,85). Son familias con pocos medios económicos ( $\bar{X}=1,76$ : de 10 a 15 puntos aproximadamente), pero con bastantes recursos para el aprendizaje $(\bar{X}=2,95$ : de 6 a 9 recursos) y un número de libros que oscila entre 40 y $120(\bar{X}=3,28)$.

Las tablas 4 y 5 muestran las frecuencias y porcentajes de cada una de las categorías de las dos variables cualitativas consideradas para la constitución de los cluster.

Tabla 4. Descriptivos de la variable titularidad del centro por conglomerados en Educación Infantil. Frecuencias y porcentajes.

\begin{tabular}{lcccccc}
\hline \multirow{2}{*}{ Categoría } & Estadístico & \multicolumn{5}{c}{ Conglomerado } \\
\cline { 2 - 7 } & & 1 & 2 & 3 & 4 & Combinados \\
\hline \multirow{2}{*}{ Pública } & Frecuencia & 0 & 220 & 531 & 466 & 1.217 \\
\cline { 2 - 7 } & Porcentaje &, $0 \%$ & $18,1 \%$ & $43,6 \%$ & $38,3 \%$ & $100 \%$ \\
\hline \multirow{2}{*}{ Concertada } & Frecuencia & 615 & 81 & 0 & 0 & 696 \\
\cline { 2 - 7 } & Porcentaje & $88,4 \%$ & $11,6 \%$ & $0 \%$ & $0 \%$ & $100 \%$ \\
\hline \multirow{2}{*}{ Privada } & Frecuencia & 28 & 3 & 0 & 0 & 31 \\
\cline { 2 - 7 } & Porcentaje & $90,3 \%$ & $9,7 \%$ & $0 \%$ & $0 \%$ & $100 \%$ \\
\hline
\end{tabular}

Tabla 5. Descriptivos de la variables nacionalidad del padre y/o la madre por conglomerados en Educación Infantil. Frecuencias y porcentajes.

\begin{tabular}{lcccccc}
\hline \multirow{2}{*}{ Categoría } & Estadístico & \multicolumn{5}{c}{ Conglomerado } \\
\cline { 2 - 7 } & & 1 & 2 & 3 & 4 & Combinados \\
\hline \multirow{2}{*}{ España } & Frecuencia & 643 & 0 & 531 & 466 & 1.640 \\
\cline { 2 - 7 } & Porcentaje & $39,2 \%$ & $0 \%$ & $32,4 \%$ & $28,4 \%$ & $100 \%$ \\
\hline \multirow{2}{*}{ Otro país } & Frecuencia & 0 & 304 & 0 & 0 & 304 \\
\cline { 2 - 7 } & Porcentaje & $0 \%$ & $100 \%$ & $0 \%$ & $0 \%$ & $100 \%$ \\
\hline
\end{tabular}

Características del tipo familiar 1 en Educación Infantil

Este primer perfil familiar representa el $33,1 \%$ de las familias que configuran la muestra de la investigación (Tabla 2). Son padres y madres de nacionalidad española
(Tabla 5), de alumnado de centros concertados y privados (Tabla 4 ).

Como muestra la Figura 1, tanto la variable "Nacionalidad del padre o de la madre", como la "Titularidad del centro" han resultado ser estadísticamente significativas para conformar 
este primer perfil familiar (valores críticos de $\quad$ Nacionalidad= 5,02).

Chi-cuadrado: Titularidad centro $=7,38$;

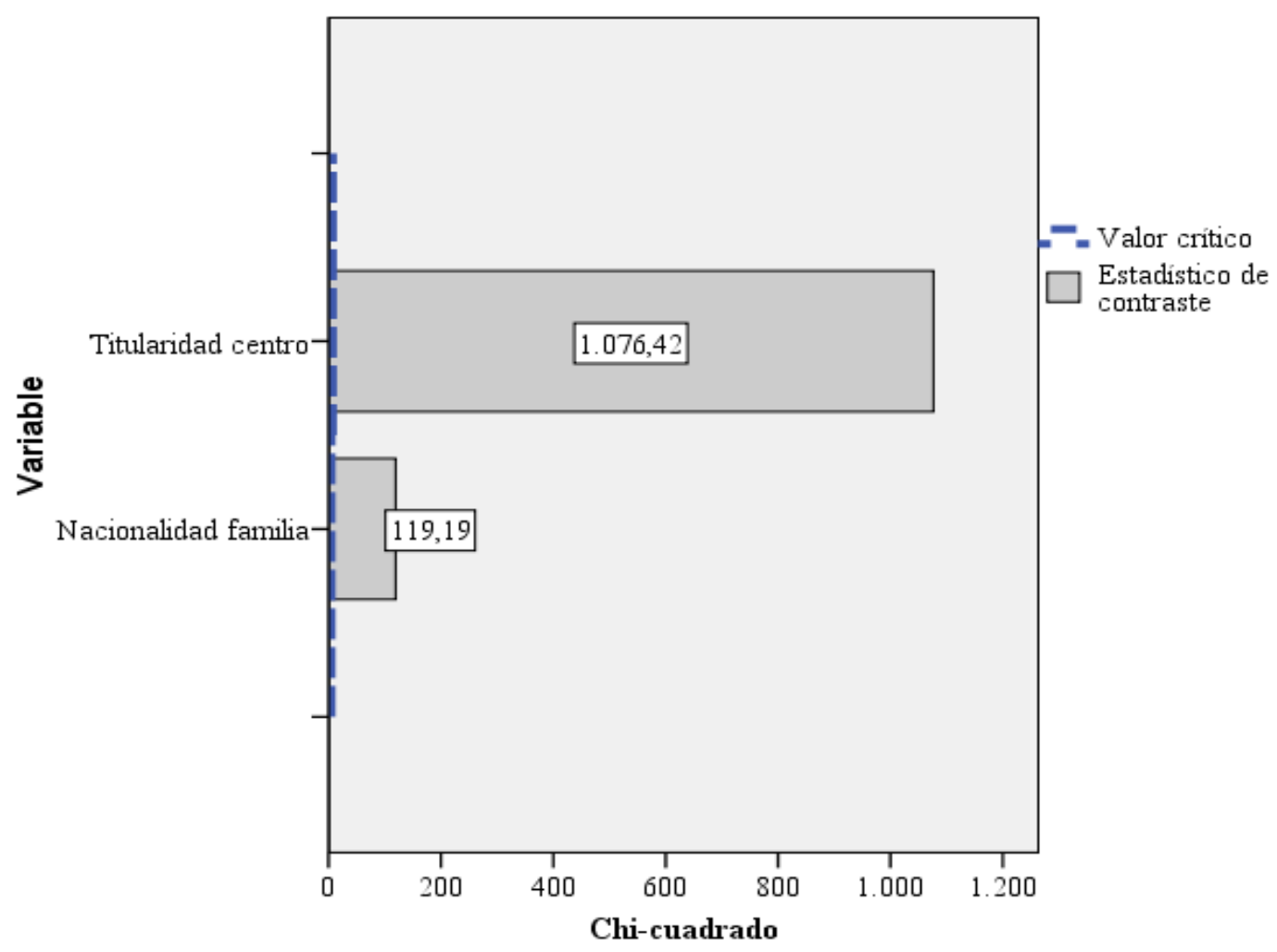

Figura 1. Importancia de las variables categóricas en el conglomerado 1.

La Tabla 3 indica que esta tipología de familias, percibe bastante alta la calidad de la comunicación con el centro educativo en el que cursan estudios los hijos ( $\bar{X}=2,75$, sobre 4); participan moderadamente en las actividades organizadas por el centro $(\bar{X}=2,29$, sobre 4); poseen bastante sentimiento de pertenencia ( $\bar{X}=3,20$, sobre 4$)$ y se implican entre bastante y mucho en la educación de los hijos desde el hogar ( $\bar{X}=3,51$, sobre 4$)$. Contrariamente a estas valoraciones, las familias que conforman este primer perfil, se implican escasamente, tanto en la AMPA del centro $(\bar{X}=2,15$, sobre 8$)$, como en el Consejo Escolar del mismo ( $\bar{X}=2,01$ sobre 8$)$. En general, de los cuatro conglomerados obtenidos, este perfil se sitúa en segunda posición en lo que respecta a la participación de padres y madres en el proceso educativo de los hijos $(\bar{X}=2,67)$.

Respecto al resto de variables numéricas, la Tabla 3 señala que este conglomerado queda conformado por padres y madres de una edad media aproximada de 43 y 41 años, respectivamente ( $\bar{X}=3,33$ y $\bar{X}=3,14$, sobre 4); los padres poseen estudios promedio algo inferiores a FP de Grado Superior $(\bar{X}=4,79$, sobre 8) y las madres han alcanzado algo más de dicho nivel educativo $(\bar{X}=5,27$, sobre 8$)$. Se trata de familias con pocos recursos materiales, ya que solo han alcanzado de 10 a 15 puntos ( $\bar{X}=1,77$, sobre 4 ), poseen bastantes recursos de aprendizaje ( $\bar{X}=3,07$, sobre 4 : de 7 a 10 recursos), así como entre 50 y 150 libros ( $\bar{X}=3,51$, sobre 6 intervalos).

En la Figura 2, se observa la relevancia significativa de 10 de las 13 variables numéricas contempladas, todas ellas con valores superiores a la media, a excepción de las referidas a la "Implicación en el Consejo Escolar" y a la "Implicación en la AMPA" (valores críticos de $\mathrm{t}$ de Student de -2,90 y $2,90)$. 


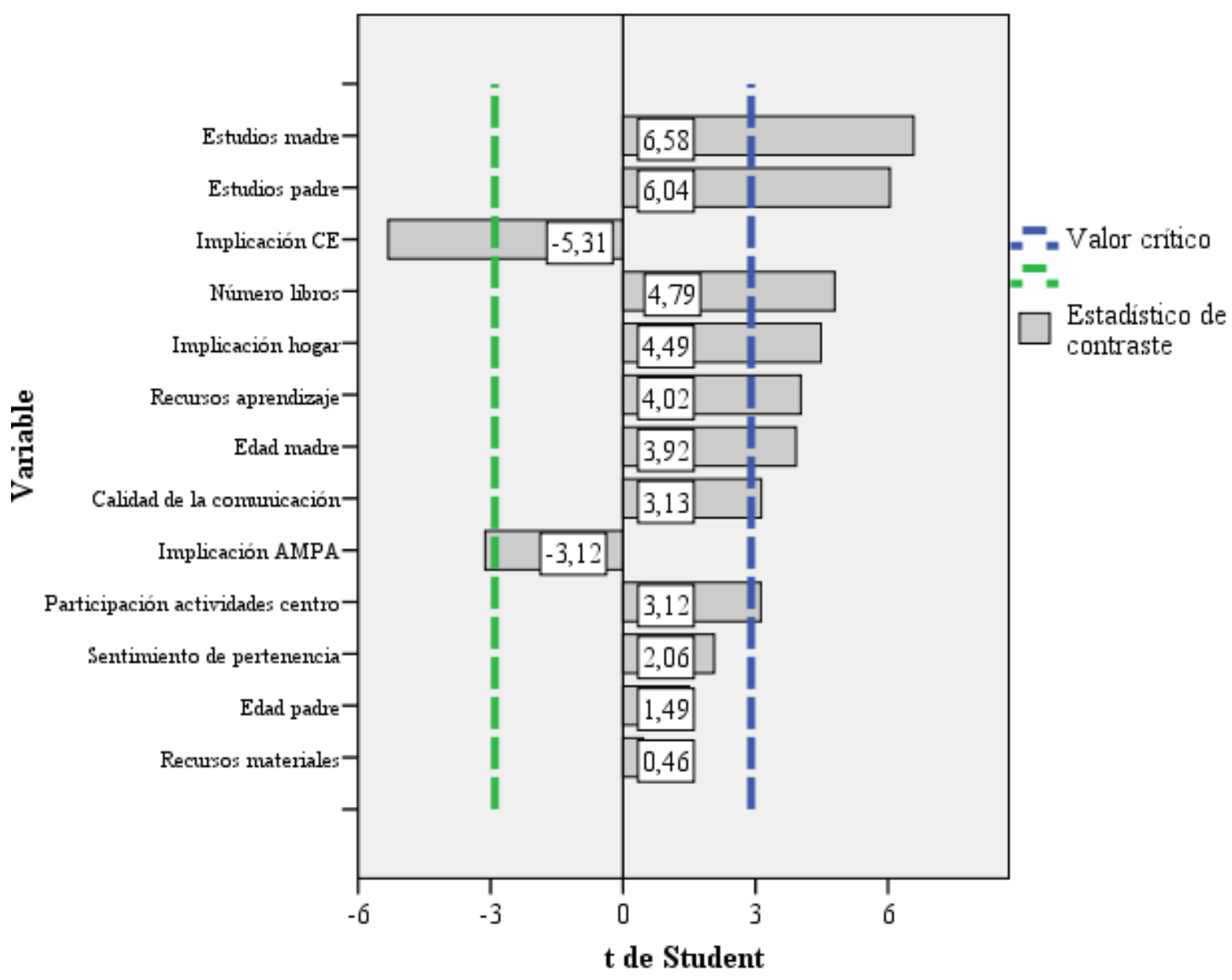

Figura 2. Importancia de las variables numéricas en el conglomerado 1.

Características del tipo familiar 2 en Educación Infantil

Este segundo perfil representa el 15,6\% de las familias del alumnado de Educación Infantil (Tabla 2). Lo conforman padres y madres con nacionalidad diferente a la española (Tabla 5), cuyos hijos e hijas cursan sus estudios mayoritariamente en centros de titularidad pública (Tabla 4).
En la Figura 3 se aprecia que las dos variables categóricas elegidas en el estudio han sido significativamente relevantes para la constitución de este segundo conglomerado en la etapa de Educación Infantil (valores críticos de $\quad$ Chi-cuadrado: $\quad$ Nacionalidad=7,38; Titularidad centro $=5,02$ ). 


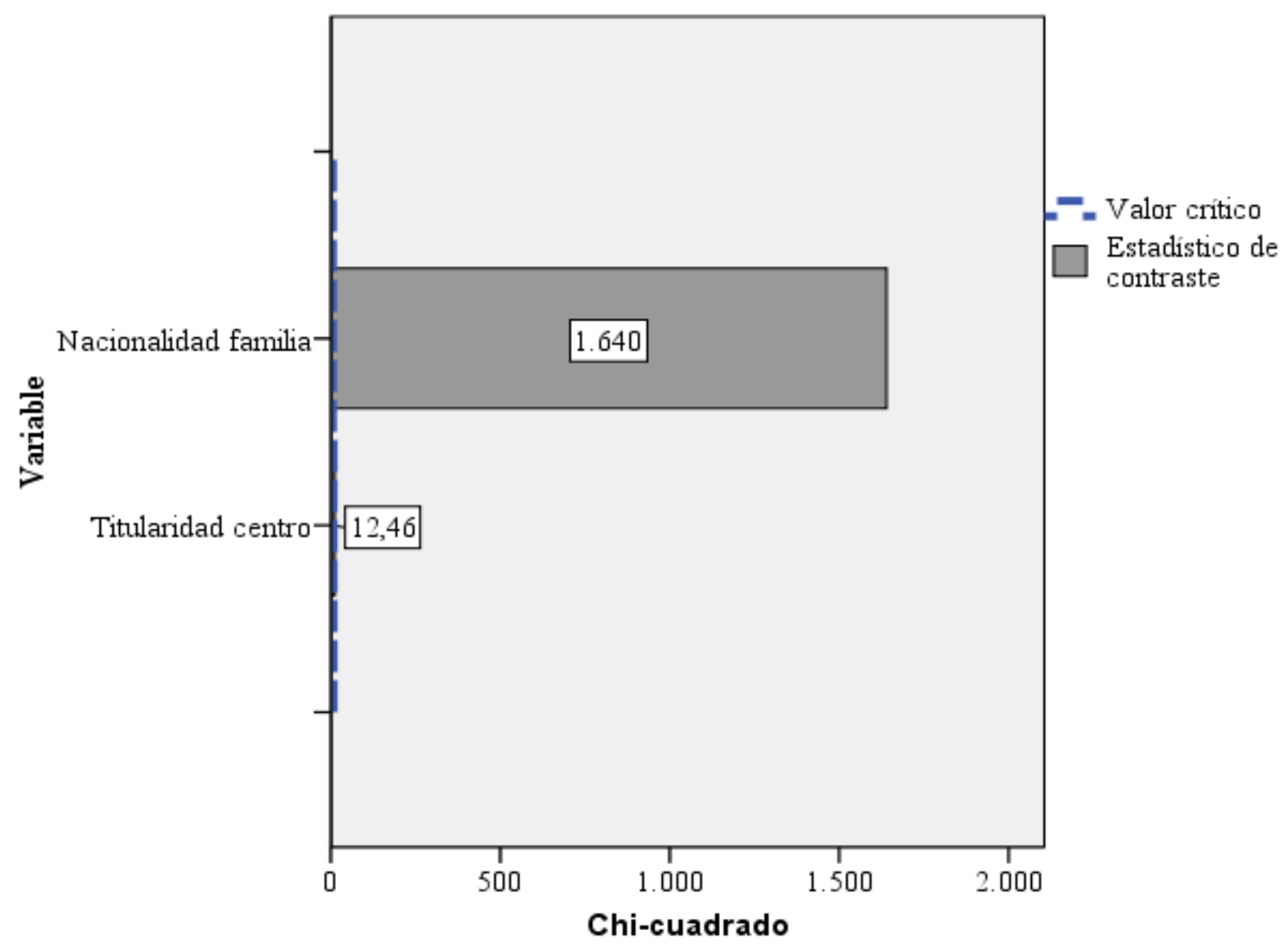

Figura 3. Importancia de las variables categóricas en el conglomerado 2.

La Tabla 3 muestra que el perfil 2 está integrado por familias que califican de moderada la calidad de comunicación con el centro $(\bar{X}=2,65$, sobre 4$)$; con poca participación en las actividades organizadas por el centro ( $\bar{X}=2,03$, sobre 4$)$; bastante sentimiento de pertenencia $(\bar{X}=3,14$, sobre 4$)$, así como de colaboración en el proceso educativo de los hijos desde el hogar $(\bar{X}=3,36$, sobre 4). Estas familias se implican poco en la AMPA ( $\bar{X}=1,93$, sobre 8$)$, y en el Consejo Escolar del centro $(\bar{X}=1,97$, sobre 8$)$. Globalmente, es la tipología que se encuentra en el tercer puesto en lo que concierne a su nivel de participación en el proceso educativo de los hijos $(\bar{X}=2,51)$, respecto al resto de conglomerados obtenidos.

En la Tabla 3 también se aprecia que este segundo perfil agrupa padres y madres de unos
43 y 40 años de edad media, respectivamente ( $\bar{X}=3,34$ y $\bar{X}=2,98$, sobre 5 intervalos); con estudios medios del padre situados en el Bachillerato o FP de Grado Medio ( $\bar{X}=4,15$, sobre 8), y de la madre entre este último nivel y FP de Grado Superior ( $\bar{X}=4,57$, sobre 8 ); son familias con escasos recursos materiales ( $\bar{X}=1,66$, sobre 4 intervalos; de 9 a 14 puntos), poseen de 5 a 8 recursos para el aprendizaje ( $\bar{X}=2,84$, sobre 4 intervalos) y de 26 a 100 libros ( $\bar{X}=2,90$, sobre 6 intervalos).

Como se observa en la Figura 4, para conformar este perfil familiar, se ha hallado significación estadística en 7 de las 13 variables numéricas contempladas en la investigación (valores críticos de t de Student de -2,91 y 2,91), todas ellas con puntuaciones inferiores a la media. 


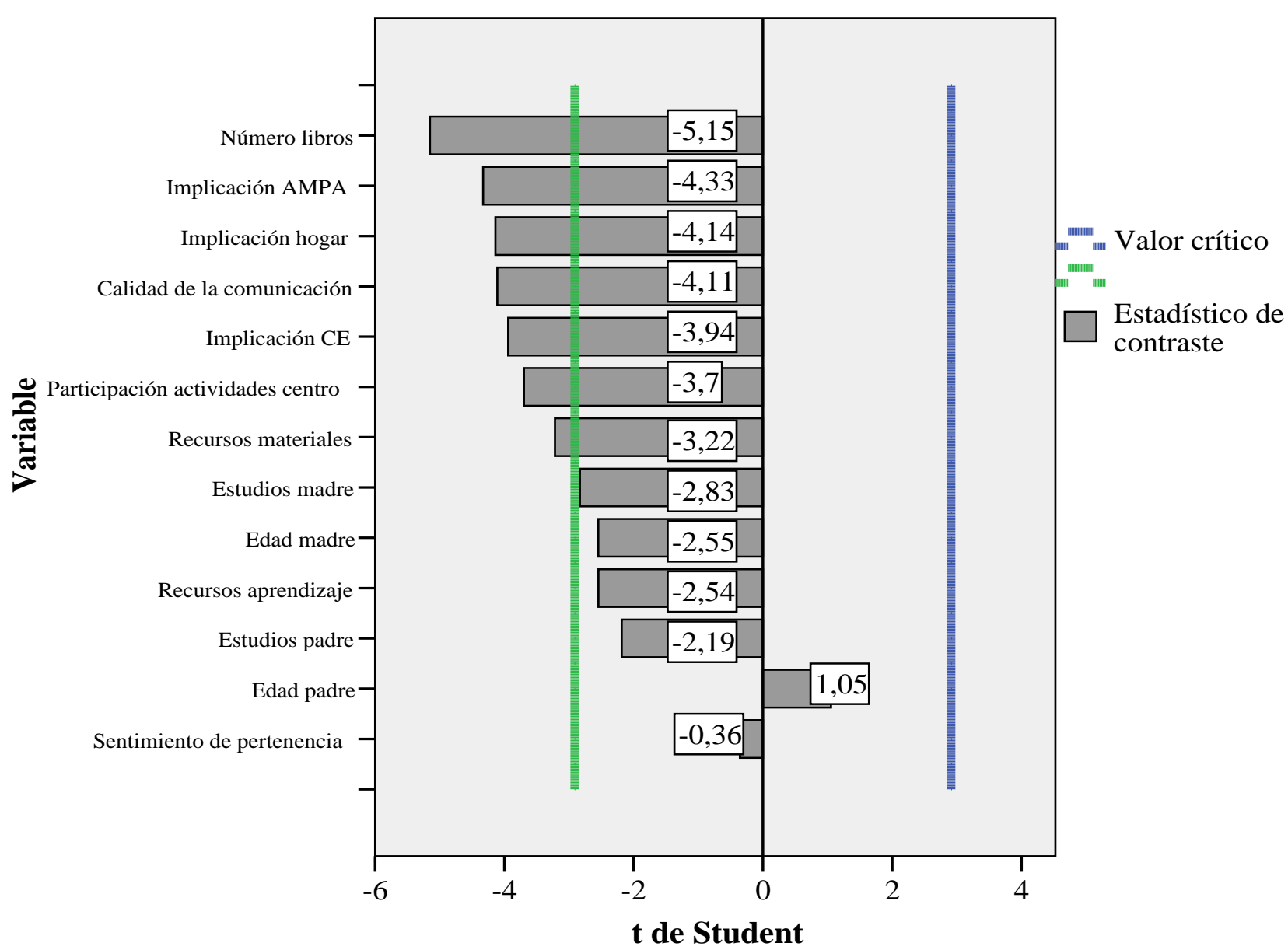

Figura 4. Importancia de las variables numéricas en el conglomerado 2.

Características del tipo familiar 3 en Educación Infantil

Este conglomerado representa el $27,3 \%$ de las familias participantes (Tabla 2). Está formado por padres y madres españoles (Tabla 5), cuyos hijos acuden a centros públicos (Tabla 4). La Figura 5 indica que tanto la variable "Titularidad del centro" como la relacionada con la "Nacionalidad de las familias", al igual que ocurre en los tipos familiares 1 y 2 , son significativamente relevantes para la constitución del conglomerado (valores críticos de Chicuadrado: $\quad$ Titularidad $\quad$ centro=7,38; Nacionalidad $=5,02$ ). 


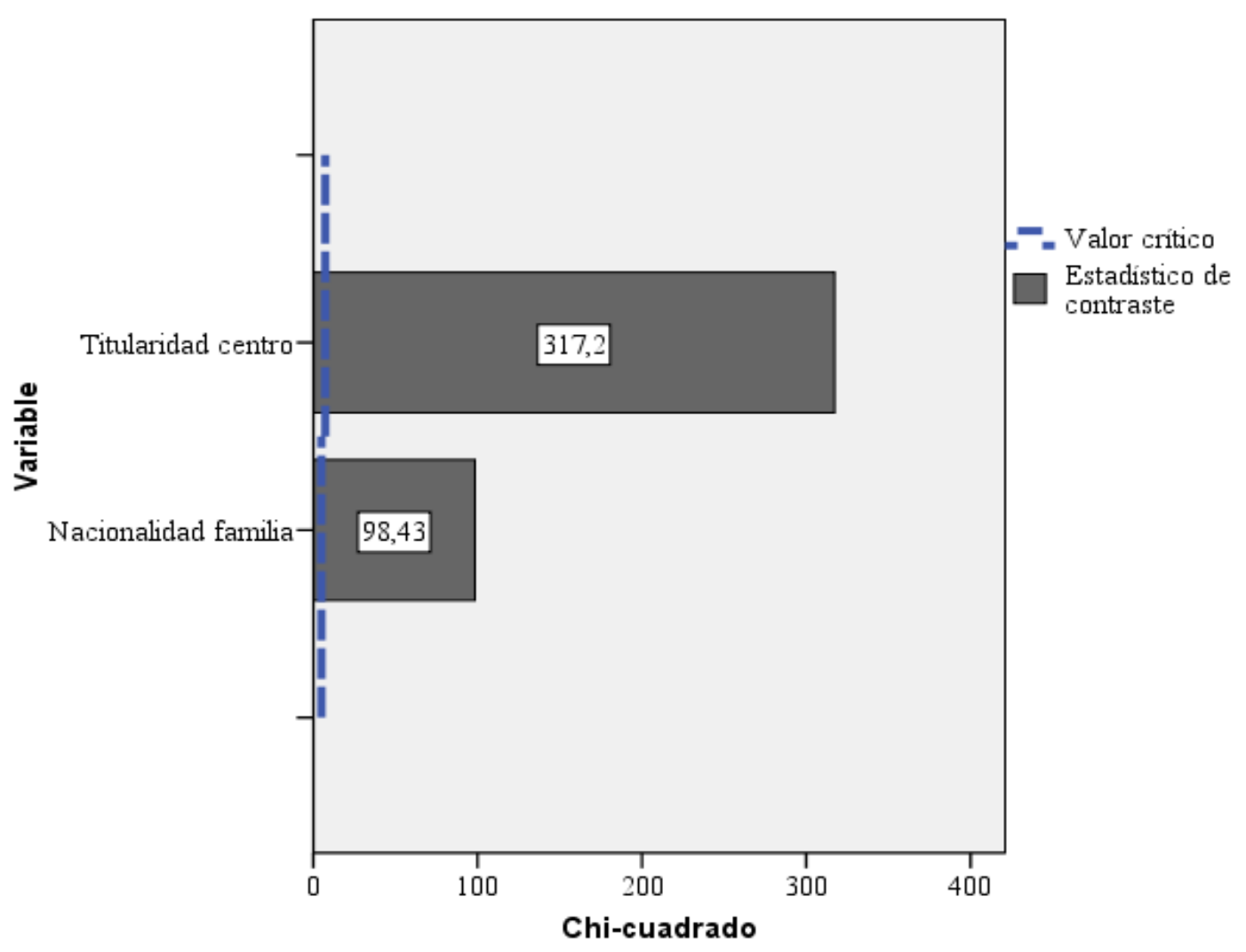

Figura 5. Importancia de las variables categóricas en el conglomerado 3.

Este cluster conforma familias con el mayor nivel de participación en la educación escolar de los hijos $(\bar{X}=3,30)$. Así, como se aprecia en la Tabla 3 , se trata de padres y madres que consideran alta la calidad de la comunicación con el centro educativo ( $\bar{X}=2,90$, sobre 4$)$; con moderada participación en las actividades organizadas por el centro $(\bar{X}=2,46$, sobre 4$)$; poseen un elevado sentimiento de pertenencia $(\bar{X}=3,36$, sobre 4$)$ y colaboran entre bastante y mucho en la educación directa de los hijos desde el hogar $(\bar{X}=3,59$, sobre 4). Estas familias se implican bastante más que las pertenecientes a los dos perfiles anteriores, en la AMPA ( $\bar{X}=3,73$, sobre 8$)$, y en el Consejo Escolar del centro $(\bar{X}=3,77$, sobre 8$)$.

En la Tabla 3 también se aprecia que este tercer perfil familiar integra los padres y madres de mayor edad, con una edad media aproximada de los primeros de 44 años $(\bar{X}=$
3,37) y de las madres de $42(\bar{X}=3,17)$; los padres casi han alcanzado estudios medios de FP de Grado Superior ( $\bar{X}=4,80$, sobre 8$)$, mientras que las madres han sobrepasado este nivel educativo $(\bar{X}=5,33$, sobre 8$)$. Si bien los recursos materiales que poseen las familias de este conglomerado continúan siendo escasos, aumenta la cantidad de los mismos $(\bar{X}=1,87$, sobre 4 intervalos; de 10 a 18 puntos); asimismo, se trata de padres y madres que disponen de entre 8 a 11 recursos de aprendizaje ( $\bar{X}=3,39$, sobre 4 intervalos) y entre 101 y 200 libros $(\bar{X}=3,69$, sobre 6 intervalos).

Asimismo, la Figura 6 muestra que las 13 variables numéricas seleccionadas han resultado ser estadísticamente significativas para la configuración del tercer tipo, todas ellas superiores a la media (valores críticos de t de Student de -2,9 y 2,9). 


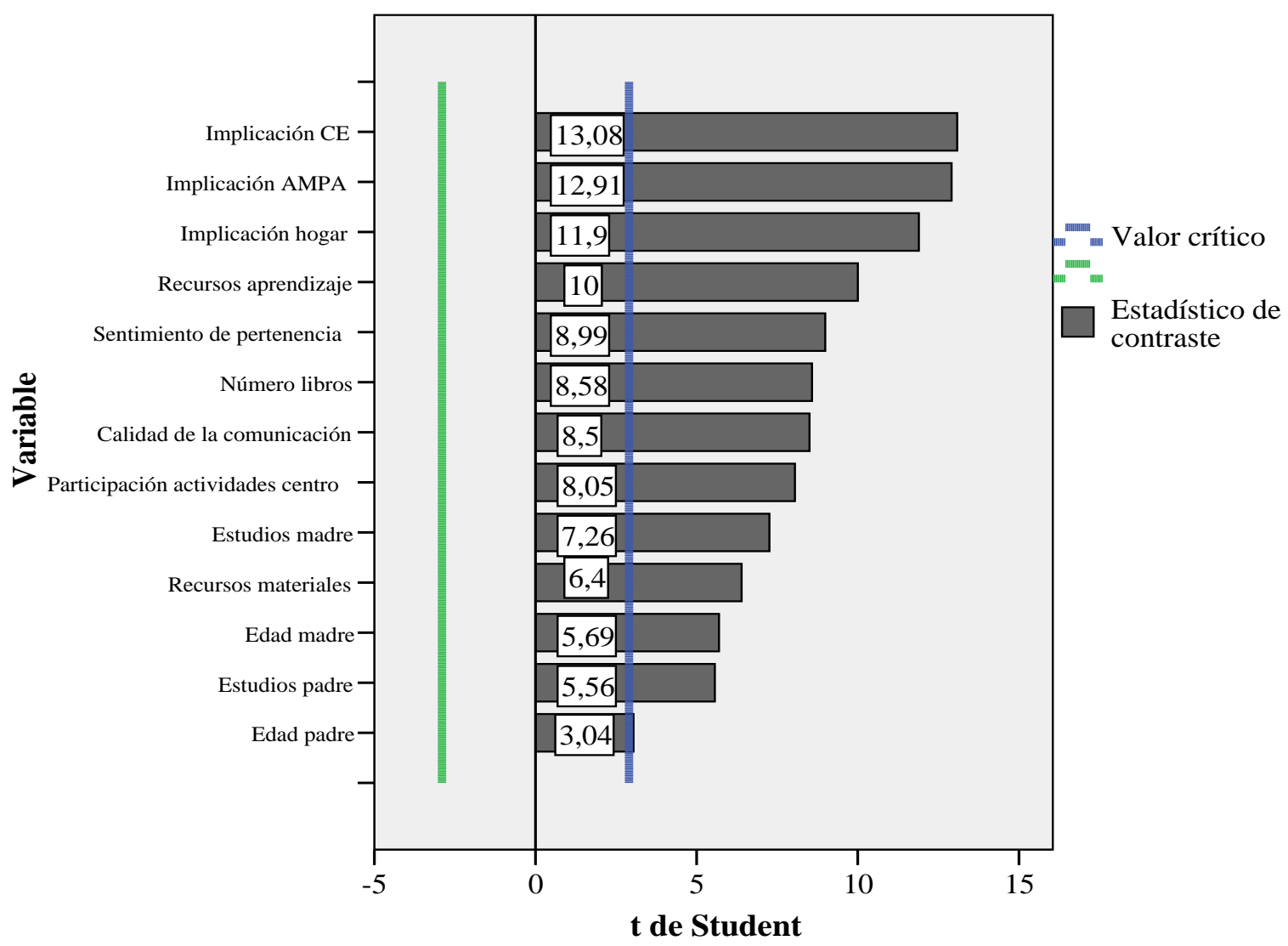

Figura 6. Importancia de las variables numéricas en el conglomerado 3.

Características del tipo familiar 4 en Educación Infantil

Este cuarto y último perfil lo configura el $24 \%$ de las familias del alumnado de Educación Infantil (Tabla 2). Se trata de padres y madres de nacionalidad española (Tabla 5), que han escolarizado a sus hijos en centros públicos (Tabla 4).
En la Figura 7 se aprecia la importancia de las variables "Titularidad del centro" y "Nacionalidad de la familia" para la constitución del conglomerado y, como en los otros tres perfiles, ambas han resultado ser estadísticamente significativas (valores críticos de Chi-cuadrado: Titularidad centro $=7,38$; Nacionalidad $=5,02$ ). 


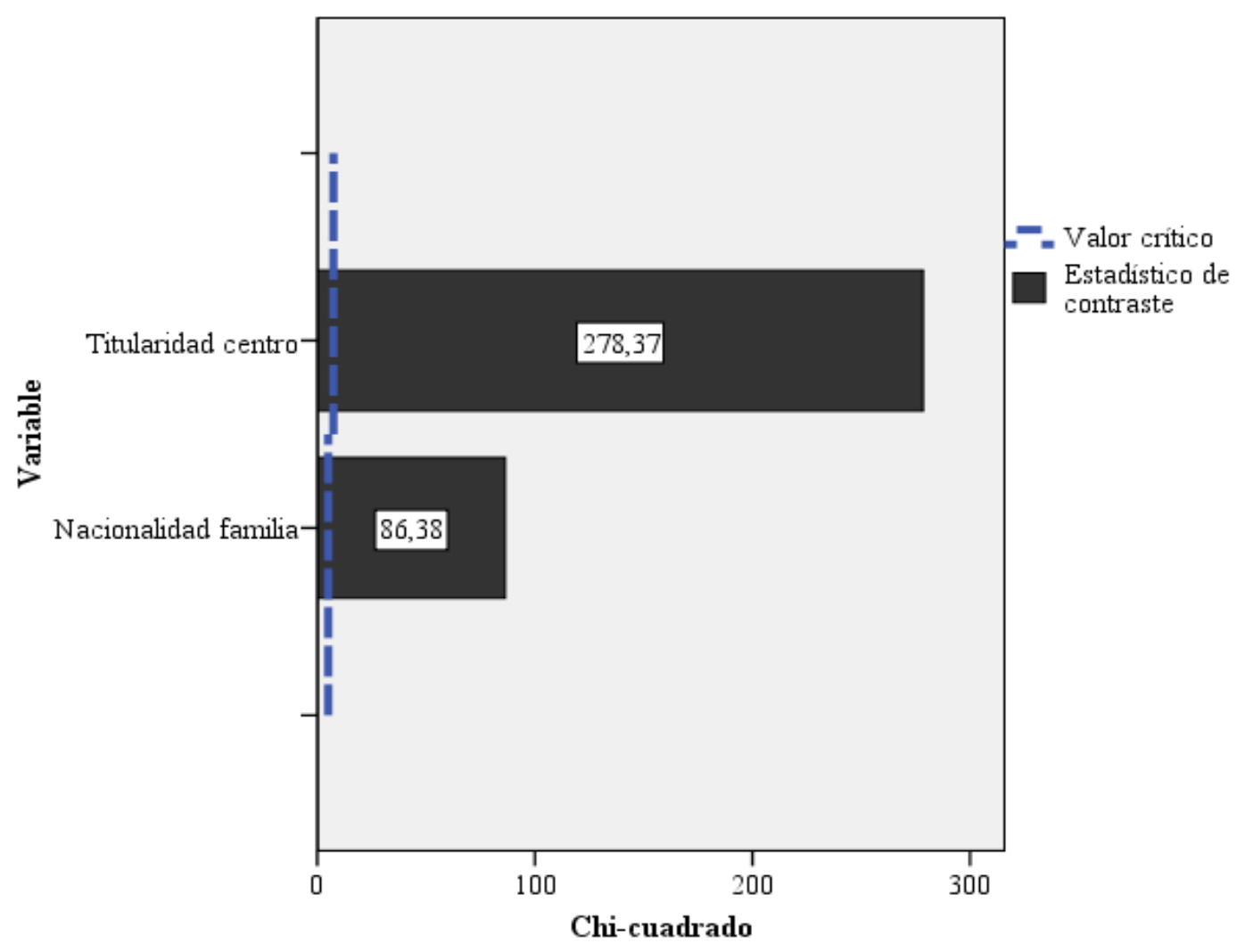

Figura 7. Importancia de las variables categóricas en el conglomerado 4.

En el polo opuesto del perfil 3, esta tipología integra familias con el nivel más bajo de participación global de los cuatro conglomerados $(\bar{X}=2,33)$. Concretamente, en la Tabla 3 se observa que son familias que perciben una moderada calidad de comunicación con el centro educativo $(\bar{X}=$ 2,58, sobre 4); con poco grado de participación en las actividades organizadas por el centro $(\bar{X}=1,86$, sobre 4$)$; bastante sentimiento de pertenencia ( $\bar{X}=2,86$, sobre 4$)$, así como el mismo grado de colaboración (bastante) en el proceso educativo de los hijos desde el hogar $(\bar{X}=3,29$, sobre 4$)$. Estas familias se implican muy poco en la AMPA ( $\bar{X}=1,62$, sobre 8$)$ y en el Consejo Escolar del centro $(\bar{X}=1,79$, sobre 8).

La Tabla 3 también muestra que este último perfil engloba padres y madres de una edad media aproximada de 41 y 39 años, respectivamente ( $\bar{X}=3,15$ y $\bar{X}=2,88$, sobre 5 intervalos), constituyendo los progenitores más jóvenes de la muestra participante; los estudios medios del padre se sitúan entre Secundaria Obligatoria y Bachillerato o FP de Grado Medio ( $\bar{X}=3,45$, sobre 8 ), y los de la madre en este último nivel ( $\bar{X}=3,91$, sobre 8$)$. Este conglomerado, aglutina familias con pocos recursos materiales ( $\bar{X}=1,70$, sobre 4 intervalos; de 15 a 25 puntos), de 4 a 8 recursos de aprendizaje $(\bar{X}=2,55$, de 4 intervalos) y de entre 20 y 50 libros $(\bar{X}=2,74$, sobre 6 intervalos).

En la Figura 8 se observa que 12 de las variables numéricas seleccionadas han resultado ser estadísticamente significativas para la configuración de este último tipo de familias, todas ellas inferiores a la media (valores críticos de $\mathrm{t}$ de Student de -2,91 y 2,91). 


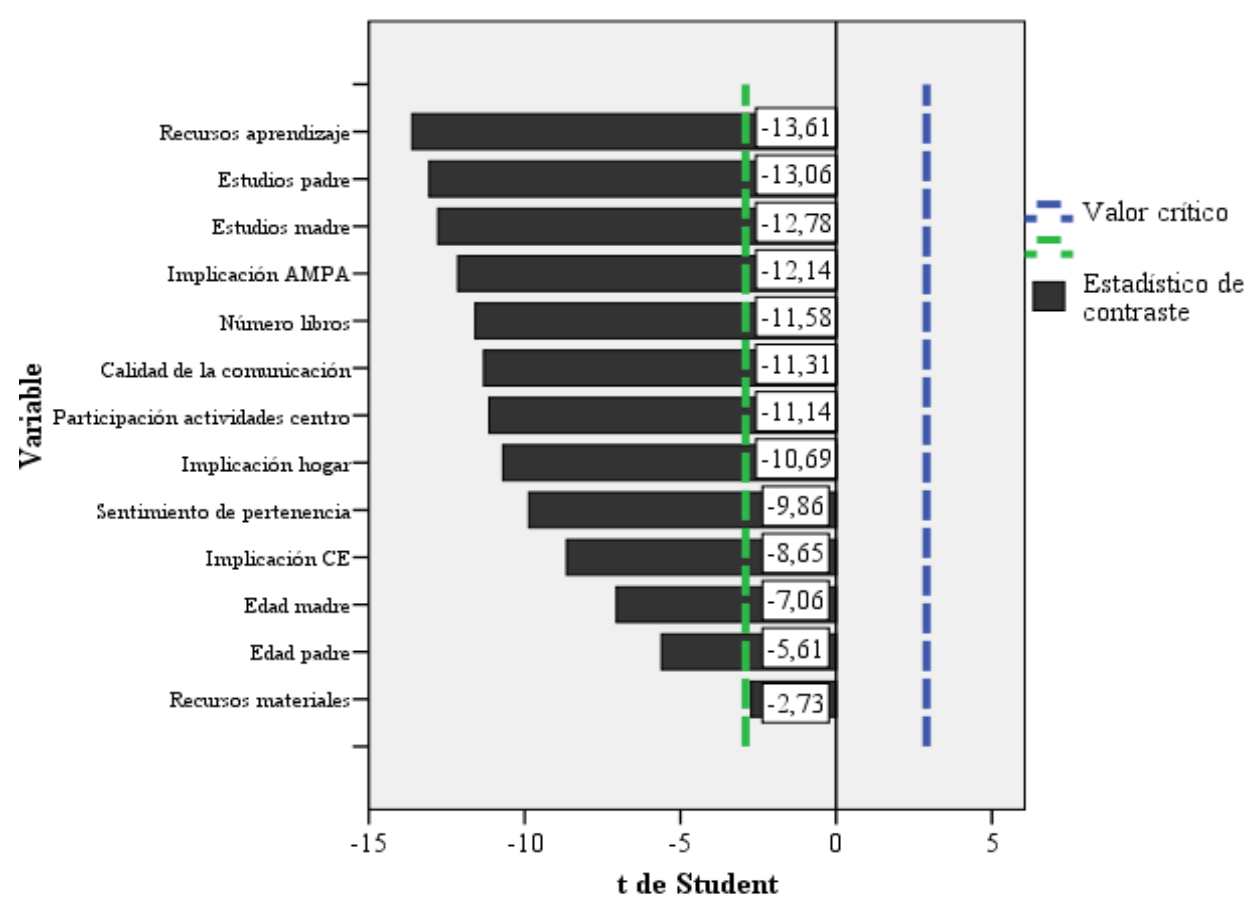

Figura 8. Importancia de las variables numéricas en el conglomerado 4.

Significación estadística y magnitud del efecto entre perfiles de participación familiar

Haciendo referencia en primer lugar a las dos variables categóricas consideradas en la investigación, tanto en lo que respecta a la "Titularidad del centro" como a la "Nacionalidad de las familias", una vez realizadas las prueba Chi-cuadrado y calculado los respectivos coeficientes de contingencia, al tomar dos a dos los cuatro perfiles familiares, se ha demostrado la existencia de diferencias significativas en todas las combinaciones posibles, así como una correlación significativa entre sendas variables y el tipo de conglomerado $(p<.05)$. No obstante, hay que hacer constar que, en cuanto a la "Titularidad del centro”, no es pertinente realizar los cálculos entre los perfiles 3 y 4, puesto que dicha variable es una constante, es decir, en los dos agrupamientos todas las familias han escolarizado a sus hijos en centros públicos. En relación a la variable "Nacionalidad de las familias", ocurre lo mismo entre los perfiles 1 y 3, 1 y 4 y 3 y 4 (todos integrados por padres y/o madres españoles).

Respecto a las variables numéricas, atendiendo primeramente a las consideradas de participación familiar, la prueba ANOVA ha mostrado la presencia de diferencias significativas a nivel global entre los perfiles hallados en este estudio ( $p<.05$ para todas las variables). No obstante, las pruebas post hoc han indicado excepciones entre algunos pares de conglomerados (Tabla 6).

Tabla 6. Ausencia de significación estadística de variables de participación familiar, entre pares de perfiles familiares

\begin{tabular}{ccc}
\hline Perfiles & Variable & Significación estadística \\
\hline \multirow{2}{*}{$1-2$} & Sentimiento de pertenencia &, 441 \\
\cline { 2 - 3 } & Implicación familiar en la AMPA &, 258 \\
\cline { 2 - 3 } & Implicación Consejo Escolar &, 973 \\
\hline \multirow{2}{*}{$2-4$} & Implicación Consejo Escolar &, 175 \\
\cline { 2 - 3 } & Implicación familiar en la AMPA &, 132 \\
\hline
\end{tabular}


En relación al resto de variables numéricas contempladas para la configuración de los perfiles familiares, de nuevo la prueba ANOVA ha puesto de manifiesto la existencia de diferencias significativas entre los mismos a nivel global ( $p<.05$ para todas las variables). Las pruebas post hoc han indicado las excepciones presentadas en la Tabla 7.

Tabla 7. Ausencia de significación estadística de variables numéricas sociodemográficas, entre pares de perfiles familiares.

\begin{tabular}{ccc}
\hline \multirow{2}{*}{ Perfiles } & Variable & Significación estadística \\
\hline \multirow{2}{*}{$1-2$} & Edad del padre &, 996 \\
\hline \multirow{2}{*}{$1-3$} & Edad del padre &, 702 \\
\cline { 2 - 3 } & Edad de la madre &, 682 \\
\cline { 2 - 3 } & Estudios padre & 1 \\
\hline $2-3$ & Estudios madre &, 891 \\
\hline \multirow{2}{*}{$2-4$} & Edad del padre &, 914 \\
\cline { 2 - 3 } & Número de libros &, 247 \\
\hline
\end{tabular}

Dado el gran tamaño de la muestra participante, además de la significación estadística, se ha calculado también la magnitud del efecto de las variables contempladas en la investigación. Para ello, se ha utilizado el valor del coeficiente de contingencia en el caso de las variables categóricas y el índice $d$ de Cohen en el caso de las variables numéricas. La interpretación del tamaño del efecto de sendas medidas se presenta en la Tabla 8 (Cohen, 1988).

Tabla 8. Interpretación del tamaño del efecto

\begin{tabular}{ccc}
\hline Interpretación & Índice $d$ & Índice $r$ \\
\hline Efecto nulo & 0 & 0 \\
\hline Efecto bajo & $\pm 0,20$ & $\pm 0,10$ \\
\hline Efecto medio & $\pm 0,50$ & $\pm 0,30$ \\
\hline Efecto alto & $\pm 0,80$ & $\pm 0,50$ \\
\hline
\end{tabular}

En lo que respecta a las variables categóricas, el coeficiente de contingencia ha superado el valor 0,3 exigido en todas las combinaciones posibles entre pares de perfiles familiares. Por lo tanto, se puede afirmar que el tamaño del efecto de las dos variables categóricas estudiadas es suficientemente fuerte como para poder aceptar una clara diferencia entre perfiles familiares. No obstante, recordemos que los cálculos referidos a la correlación existente entre los conglomerados 3 y 4, en lo que respecta a la “Titularidad del centro”, así como entre los cluster 1 y 3, 1 y 4 y 3 y 4, relativos a la "Nacionalidad de las familias", no han sido realizados porque sendas variables constituyen una constante en los agrupamientos indicados.

En relación a las variables numéricas, atendiendo en primer lugar a las consideradas variables de participación familiar, en la Tabla 9 se muestra el valor del índice $d$ de Cohen (tamaño del efecto), considerando las distintas combinaciones entre pares de conglomerados que resultaron ser estadísticamente significativas. 
Tabla 9. Tamaño del efecto de variables de participación familiar entre pares de perfiles familiares con significación estadística

\begin{tabular}{lcccccc}
\hline \multirow{2}{*}{ Variable } & \multicolumn{7}{c}{ Índice $d$ de Cohen } \\
\cline { 2 - 7 } & Perfiles & Perfiles & Perfiles & Perfiles & Perfiles & Perfiles \\
& $1-2$ & $1-3$ & $1-4$ & $2-3$ & $2-4$ & $3-4$ \\
\hline Calidad de la comunicación &, 365 &, 246 &, 600 &, 597 &, 162 &, 870 \\
Participación actividades &, 334 &, 332 &, 598 &, 562 &, 245 &, 848 \\
Sentimiento de pertenencia & &, 275 &, 549 &, 368 &, 431 &, 849 \\
Colaboración hogar &, 419 &, 271 &, 682 &, 674 &, 182 & 1,001 \\
Implicación AMPA & &, 713 &, 327 &, 831 & & 1,093 \\
\hline Implicación CE & &, 900 & &, 816 & &, 980 \\
\hline
\end{tabular}

Como se aprecia (Tabla 9), existe una diferencia claramente evidente entre las tipologías familiares 3 y 4 en lo que respecta a todas las variables consideradas de participación familiar. Esta diferencia también se observa entre los perfiles 2 y 3, excepto en relación a la variable "Sentimiento de pertenencia”, así como también entre los perfiles 1 y 4, a excepción de la relativa a las variables "Implicación en la AMPA" y, por supuesto "implicación en el Consejo Escolar". En lo que respecta a estas dos últimas variables, han sido las únicas en las que las diferencias entre los perfiles 1 y 3 pueden considerarse relevantes. Por último, entre los conglomerados 1 y 2, así como entre el 2 y el 4 no se aprecian claras diferencias, puesto que el índice $d$ no ha alcanzado el efecto medio en ninguna de las variables.
En cuanto a las variables numéricas sociodemográficas, la Tabla 10 indica una magnitud del efecto igual o superior al típico entre los perfiles 1 y 4, así como entre el 3 y 4, excepto en lo que concierne a las variables "Edad del padre" y "Recursos materiales". Asimismo, las diferencias son suficientemente amplias entre los perfiles 2 y 3 en relación a las variables en las que se ha obtenido significación estadística, a excepción de las variables "Edad de la madre" y "Estudios del padre”. Entre los perfiles 1 y 2 únicamente se ha hallado una diferencia clara en lo relativo a la variable "Número de libros" (reduciendo el índice $d$ a 1 decimal). No se aprecia la magnitud del efecto requerida entre los perfiles 1 y 3 , ni entre los perfiles 2 y 4 en ninguna de las variables.

Tabla 10. Tamaño del efecto de variables numéricas sociodemográficas entre pares de perfiles familiares con significación estadística

\begin{tabular}{ccccccc}
\hline Variable & \multicolumn{7}{c}{ Índice $d$ de Cohen } \\
\hline & Perfiles & Perfiles & Perfiles & Perfiles & Perfiles & Perfiles \\
& $1-2$ & $1-3$ & $1-4$ & $2-3$ & $2-4$ & $3-4$ \\
\hline Edad del padre & & &, 319 & &, 299 &, 400 \\
Edad de la madre &, 306 & &, 503 &, 381 &, 183 &, 589 \\
Estudios del padre &, 365 & &, 813 &, 370 &, 256 &, 818 \\
Estudios de la madre &, 401 & &, 900 &, 465 &, 397 &, 907 \\
Número de libros &, 489 &, 166 &, 700 &, 698 & &, 895 \\
Recursos de aprendizaje &, 297 &, 251 &, 760 &, 548 &, 410 & 1,062 \\
\hline Recursos materiales &, 199 &, 242 &, 145 &, 448 & &, 376 \\
\hline
\end{tabular}




\section{Discusión}

La participación de las familias en los centros escolares es bastante variada, con distintas intensidades, dependiendo de las características sociodemográficas esenciales de las familias. A pesar de las concreciones legislativas respecto a los derechos de participación, autores como Navaridas y Raya (2012) afirman que 'la realidad no refleja una participación activa y significativa de los padres en la vida de los centros' (p.239). En este sentido, en general, las familias de los conglomerados 2 y 4, denotan bajos niveles de participación en la vida de los centros escolares, confirmando de este modo, que en ocasiones, las relaciones entre familia y centro educativo se caracterizan por 'la crónica de un desencuentro’ (Fernández Enguita, 1993), por el desapego (Bolívar, 2006), por la aparente contemplación de objetivos diferentes (Maestre, 2009), así como por una comunicación imprecisa e ineficaz que contribuye a considerar dicha relación como un desafío educativo a plantearse (García Bacete, 2003). Según Collet y Tort (2008), el alejamiento que existe a veces entre familia y escuela tiene su origen en la diferencia estructural de las dos instituciones: modernidad versus postmodernidad.

No obstante, en la diversidad de tipologías familiares evidenciadas, la impresión que nos ofrecen los resultados obtenidos en nuestro trabajo puede que vaya por otros derroteros, ya que más de la mitad de los participantes cuyos hijos están cursando Educación Infantil, incorporadas a los conglomerados 1 y 3, muestran una implicación medio-alta en los centros educativos. Todo ello denota que la participación conjunta de las dos instituciones a partir de objetivos compartidos no es uniforme, no se da siempre de una manera única, pudiendo afirmar que las familias evolucionan en la forma de interacción con el centro y con el profesorado. Según García Bacete (2003) los padres, como clientes, pueden adoptar, de forma general, dos posiciones extremas, o bien lo dejan todo en manos del maestro (por delegación inconsciente o porque el profesor es el profesional y para ello se le paga), o bien consideran legítimo participar en la educación de los hijos y sienten perder el control sobre los mismos. Sin embargo, en la especificidad de la participación también se ha observado una evolución positiva de las familias respecto a la comunicación en la vida escolar, más diversificada en lo que respecta a las vías de comunicación, a pesar de la tendencia predominante a tratar aspectos negativos tanto conductuales, actitudinales y de logros académicos, y a que los encuentros sean iniciados en mayor medida por el profesorado, aunque ya empiezan a ser demandadas algunas tutorías por parte de las familias (Gomariz et al., 2008).

Según lo expuesto, lo relevante no es la diferencia en los momentos cercanos en la escolaridad, limitándose a interactuar en etapas iniciales y quedando descolgados posteriormente, pero sí el modo de interactuar de forma continuada de las familias con el centro en el recorrido total de hijos (MartínezGonzález \& Álvarez-Blanco, 2005). En el caso de las familias, este recorrido ya no presenta una única bifurcación bipolar que se reduce a la existencia o no de participación, sino una diversidad de caminos que se abren a la familia en función de las elecciones de participación y modos de implicación. Atendiendo a los resultados obtenidos, podemos identificar cuatro perfiles donde ciertas variables sociodemográficas como el origen nacional, la titularidad del centro, los estudios de los padres y madres, su edad, los recursos de aprendizaje, etc. se muestran asociadas con diversos niveles de participación en el centro, calidad de la participación, sentido de pertenencia o participación indirecta a través de AMPA y Consejo Escolar.

El primer tipo de familias lo hemos denominado participativo no normativo. Son familias de nacionalidad española cuyos hijos e hijas acuden a centros concertados y privados, con un nivel de estudios medio-alto y escasos recursos económicos, aunque gran 
parte de éstos los invierten en el aprendizaje de los discentes. La denominación otorgada a este perfil se debe a que el mismo está integrado por padres y madres que participan activamente en la educación de los hijos, pero a la hora de colaborar a través de los órganos de participación formal, muestran una reducida implicación tanto en la AMPA como en el Consejo Escolar del centro. Santos \& Lorenzo (2009) consideran que la inserción de las familias en la AMPA es valorada por las propias familias como poco satisfactoria, siendo muy baja la tasa de padres y madres que mantienen una relación activa con ésta.

El segundo de los tipos familiares, que podríamos denominar no participativo de nacionalidad no española, se caracteriza por integrar familias de nacionalidad diferente a la española de, al menos, uno de los progenitores. Se trata de padres y madres que matriculan a sus hijos e hijas fundamentalmente en centros públicos y que disponen de recursos económicos bajos o modestos. El nivel de implicación directa de estas familias en las actividades del centro es escaso, unido a un grado de implicación indirecta a través de la participación en AMPA y Consejo Escolar de centro aún inferior al anterior perfil. Es un perfil difuso, ya que se encuentra definido por algo menos de la mitad de las variables consideradas en el estudio.

Según Santos y Lorenzo (2009), las familias en las que alguno o ambos progenitores no es de nacionalidad española mantienen comportamientos de distanciamiento con el centro. Alguno de los aspectos que evidencian el alejamiento de estas familias con el centro educativo se ve reflejado en que más de la mitad de éstas afirman no conocer o conocer poco a los tutores y resto del profesorado de sus hijos e hijas. Otros estudios también han puesto de manifiesto que las familias inmigrantes participan en reuniones escolares y actividades del centro escasamente, con menor frecuencia que las familias autóctonas (Hernández, Gomariz, Parra \& García-Sanz, 2016). También Martínez-González, Rodríguez y Gimeno (2010) indican una medio-alta participación de estas familias en la AMPA, en lo que a inscripción se refiere (76\% de familias pagan las cuotas de la AMPA), pero una escasa implicación en su organización (78\% no colabora) y participación en actividades (lo hace menos de la mitad de las mismas). Asimismo, González Falcón (2007) destaca que una de las principales demandas de los docentes respecto a las familias inmigrantes se centra en reclamar su implicación en la etapa de Educación Infantil, pues muchas de estas familias proceden de países en los que la escolarización en edades tempranas no está generalizada (África Subsahariana, Magreb, Latinoamérica, etc.), produciéndose además la comunicación principal a través de la madre (Bueno \& Belda, 2005; Santos, Esteve, Ruiz \& Lorenzo, 2004).

Aunque ocupando el tercer lugar respecto a las cuatro tipologías de familias halladas en la presente investigación, en este segundo perfil, el sentimiento de pertenencia al centro mostrado por estas familias es bueno, lo cual indica que las familias inmigrantes están motivadas para participar en la vida del centro, pero no saben cómo hacerlo. En este sentido, coincidimos con Reparaz y Naval (2014), quienes afirman que para participar no es suficiente con disponer de voluntad e iniciativa; además de querer, se debe saber y poder participar.

Al tercer tipo de familia lo hemos denominado participativo normativo. Se trata de padres y madres españoles que han matriculado a sus hijos e hijas en centros públicos, tienen la mayor edad y un alto nivel de estudios. Aunque poseen más recursos humanos que el resto de perfiles familiares, éstos siguen siendo escasos, si bien destinan gran parte de los mismos a la educación de sus descendientes. Se caracterizan por ser los que más participan en las actividades del centro y los que se implican más en la AMPA y en el Consejo Escolar, aunque dicha implicación se queda en moderada. Consideran el centro como algo suyo, es decir, su sentimiento de 
pertenencia al centro es alto, siendo el más elevado de todos los perfiles.

El cuarto y último tipo de familias responde al perfil no participativo de nacionalidad española, cuyos hijos e hijas asisten exclusivamente a centros públicos. Son los padres y madres más jóvenes, presentan el más bajo nivel de estudios de todas las tipologías, así como el menor número de recursos que destinan al aprendizaje escolar. Su sentimiento de pertenencia, nivel de participación directa en el centro e implicación en la AMPA y en el Consejo Escolar son los más bajos de todos los perfiles de familias. Aunque coincidimos con Garreta (2008) en afirmar que la implicación familiar varía en función del origen nacional o no de los progenitores, nuestros resultados difieren del citado estudio al encontrar que este cuarto perfil de familias, siendo autóctonos, son los que menor grado de participación en la escuela presentan.

Entre los aspectos esenciales que nos pueden ayudar a explicar las razones de la implicación de las familias, entendemos que la nacionalidad, el nivel de estudios, la titularidad del centro, los recursos de aprendizaje y el número de libros son, en general, los más definitorios y con mayor capacidad predictiva para estimar la participación de las familias en los centros. Sin ser un rasgo exclusivo y excluyente de ellas, las familias con mayor nivel de formación encuentran la educación de sus hijos e hijas como algo en lo que se deben involucrar en cuanto a acompañamiento, seguimiento e implicación. Y las familias cuyo nivel de formación es más bajo se sitúan en los menores niveles de participación e implicación en la vida escolar. En este sentido, coincidimos con Martínez-González et al. (2000) en la necesidad de que los centros estimulen la comunicación más directa de los padres y madres con menores niveles de estudios, para que esto pueda facilitar su implicación en el apoyo educativo y seguimiento del aprendizaje desde edades tempranas.

También los recursos de aprendizaje, incluyendo el número de libros se encuentran en consonancia con los niveles de participación familiar. Respecto al papel de los recursos económicos, en nuestro estudio esta variable no ha sido significativa para configurar los perfiles 1 y 4 . En otras investigaciones los resultados son difusos. Por un lado, encontramos numerosos estudios que coinciden en señalar la relación que existe entre las posibilidades económicas de las familias como uno de los inputs familiares fuertes condicionantes de los resultados de la escuela (Santín, 2001). Así, Bolívar (2006) reconoce que la participación de las familias suele ser escasa, sobre todo en aquellos contextos socioeconómicos empobrecidos, curiosamente donde se requiere mayor implicación. En estas condiciones, son muchas las dificultades que presenta la familia para poder implicarse: la ausencia de los progenitores del núcleo familiar por dedicar interminables jornadas al trabajo, la carencia de un modelo de educación familiar, la desconfianza en la educación como vía para un futuro mejor, las alternativas poco favorables que ofrece el contexto, entre otras. $\mathrm{Al}$ contrario, Domínguez y Fernández (2007) concluyen que el nivel socioeconómico de las familias no influye en los aspectos relacionados con su participación. Feito (2007) defiende que a pesar de no existir diferencias significativas en la participación en función de los recursos económicos según la ubicación del centro, las familias de centros situados en barrios desfavorecidos se implican menos en la educación de sus hijos que las familias de centros situados en barrios de clase media-alta. Lo que evidencia la necesidad de establecer compromisos de actuación conjunta entre familia y escuela en los contextos vulnerables.

Respecto a la diferencia entre los cuatro tipos de participación familiar, se ha comprobado que la ausencia de significación estadística entre perfiles afecta fundamentalmente a la segunda tipología, coincidiendo con el conglomerado menos definido de los cuatro. En cuanto a la magnitud de las diferencias que han resultado ser significativas, se aprecia clara distancia 
entre los perfiles 1-4, 2- 3 y 3-4. Entre el resto de perfiles las diferencias son más difusas.

Así pues, en relación a la hipótesis de investigación, se puede afirmar que, si bien se ha demostrado la existencia de agrupamientos de familias que giran en torno a una serie de variables tanto relacionadas con la participación familiar, como de carácter sociodemográfico, la diferencia o distancia entre algunos perfiles no queda totalmente definida.

Situarnos en Educación Infantil, la etapa en la que tradicionalmente se mantiene una relación y comunicación más fluida entre familia y escuela, no garantiza, por sí misma, una participación e implicación familiar de calidad en la educación de sus hijos e hijas. Es preciso diseñar programas de intervención dirigidos a la formación de padres y madres, que prioricen la inclusión de grupos de familias con dinámicas de poca participación, de tal forma que todas ellas puedan formar parte activa de redes colaborativas con los centros (Dabas, 2005; Parra, Ceballos, Fontana \& Bartau, 2012). En definitiva, se necesitan propuestas y programas inclusivos que potencien la democratización efectiva y que propicien el compromiso de todas las familias con la educación escolar (Buarque, 2012; Instituto de Formación del Profesorado, Investigación e Innovación Educativa, 2011).

\section{Referencias}

Azpillaga, V., Intxausti, N. \& Joaristi, L. (2014). Implicación de las familias en los centros escolares de alta eficacia en la Comunidad Autónoma Vasca. Bordón, 66(3), 27-37. doi: http://doi.org/10.13042/Bordon.2014.66302

Bolívar, A. (2006). Familia y escuela: dos mundos llamados a trabajar en común. Revista de Educación, 339, 119-146.

Buarque, M.B. (2012). La participación de los consejos escolares para la gestión democrática de la escuela pública. Educar, 48(2), 285-298. doi: http://doi.org/10.5565/rev/educar.26
Bueno, J.R. \& Belda, J.F. (2005). Familias inmigrantes en la escuela. Discursos de los agentes educativos. Valencia: Universidad de Valencia.

Cohen, J. (1988) (2a. ed.). Statistical Power Analysis for the Behavioral Sciences. New York: Academic Press.

Collet, J. \& Tort, A. (2008). Espacios de participación. Cuadernos de Pedagogía, 378, 57-60.

Consejo Escolar del Estado (2014). La participación de las familias en la educación escolar. Madrid: Ministerio de Educación, Cultura y Deporte.

Dabas, E. (2005). Redes sociales, familias y escuela. Buenos Aires: Paidós.

De Vellis, R.F. (2003) (2 ${ }^{\text {a }}$ ed.). Scale development: Theory and applications. Thousand Oaks CA: Sage.

Declaración Universal de los Derechos del Niño (1959). Aprobada por la ONU el 20 de noviembre de 1959. Consultado en, http://www.oas.org/dil/esp/Declaraci\%C3\%B 3n\%20de\%20los\%20Derechos\%20del\%20Ni \%C3\%B10\%20Republica20Dominicana.pdf

Domínguez, E. \& Fernández, L. (2007). Familia y escuela. En C. Benso \& C. Pereira (Coords.), Familia y escuela: El reto de educar en el siglo XXI (pp.109-144). Ourense: Concellería de Educación.

EasyCalculation.com (s.f.). Calculadora del tamaño del efecto. Consultado en, https://www.easycalculation.com/es/statistics leffect-size.php

Escudero, J. M. (2006). Realidades y respuestas a la exclusión educativa. En J.M. Escudero \& J. Sáez (Coords.), Exclusión Social, Exclusión Educativa (pp. 69-121). Murcia: DM/ICE, Universidad de Murcia.

Feito, R. (2007). Balance de la participación de los padres en los consejos escolares de centro. Participación Educativa, 4, 4-15.

Fernández Enguita, M. (1993). La profesión docente y la comunidad escolar: Crónica de un desencuentro. Madrid: Morata. 
Fullan, M. \& Stiegielbauer, S. (2003). El cambio educativo. Guía de planeación para maestros. México: Trillas.

García-Bacete, F.J. (2003). Las relaciones escuela-familia: un reto educativo. Infancia y Aprendizaje, 26(4), 425-437. doi: http://doi.org/10.1174/021037003322553824

Garreta, J. (2008). La participación de las familias en la escuela pública. Las Asociaciones de Madres y Padres del Alumnado. Madrid: CEAPA.

Gaviria, J.L. (2014). Prólogo. En Consejo Escolar del Estado (Coord.), La participación de las familias en la educación escolar (pp.13-18). Madrid: Ministerio de Educación, Cultura y Deporte.

Gomariz, M.A., Parra, J., García-Sanz, M.P., Hernández, M.A. \& Pérez, J. (2008). La comunicación entre la familia y el centro educativo. Murcia: Consejo Escolar de la Región de Murcia. Consejería de Educación, Ciencia e Investigación.

González Falcón, I. (2007). La participación de las familias inmigrantes en la escuela: necesidades de orientación y formación. XXI Revista de Educación Universidad de Huelva, 9, 155-169.

Hernández, M.A., Gomariz, M.A., Parra, J. \& García-Sanz, M.P. (2016). Familia, inmigración y comunicación con el centro escolar: un estudio comparativo. Educación XX1， 19(2), 127-151. doi: http://doi.org/10.5944/educxx1.16457

Instituto de Formación del Profesorado, Investigación e Innovación Educativa (2011). Actuaciones de éxito en las escuelas europeas. Colección Estudios Creade, $\mathrm{n}^{\circ} 9$. Ministerio de Educación. Madrid: Secretaría de Estado de Educación y Formación Profesional.

Maestre, A. (2009). Familia y Escuela. Los pilares de la educación. Innovación y Experiencias Educativas, 14. Consultado en, http://www.csi-csif.es/andalucia/mod_ensecsifrevistad_14.html
Martínez-González, R.A., Pereira, M., Rodríguez, B., Peña, A., Martínez, R., García González, M.P., Donaire, B., Álvarez, A.I. \& Casielles, B. (2000). Dinamización de las relaciones familia-centro escolar a través de la formación del profesorado en este campo de actuación. REOP, 11(19), 107-120. doi: http://doi.org/10.5944/reop.vol.11.num.19.20 00.11326

Martínez-González, R.A., Rodríguez, B. \& Gimeno, J.L. (2010). Áreas de cooperación entre los centros docentes y las familias, Estudio de caso. Educatio Siglo XXI, 28(1), 127-156.

Martínez-González, R.A. \& Álvarez-Blanco, L. (2005). Fracaso y abandono escolar en educación secundaria obligatoria: Implicación de la familia y los centros educativos. Aula Abierta, 85, 127-146.

Murillo, F.J. (2005). La investigación sobre eficacia escolar. Barcelona: Octaedro.

Navaridas, N. \& Raya, E. (2012). Indicadores de participación de los padres en el sistema educativo: un nuevo enfoque para la calidad educativa. Revista Española de Educación Comparada, 20, 223-248. doi: http://doi.org/10.5944/reec.20.2012.7599

Paniagua, G. (2009). El desarrollo de la Educación Infantil: un crecimiento costoso. CEE participación Educativa, 12 (monográfico Una mirada a la Educación Infantil), 20-34.

Parra, J., Ceballos, E., Fontana, M. \& Bartau, I. (2012). Familia y Educación en una sociedad en red. En C. Jiménez, J.L. García, B. Álvarez \& J. Quintanal (Coords.), Investigación y Educación en un mundo en red (pp. 95-120). Madrid: McGraw-Hill.

Peñalver, R. (2009). El Plan Educa3, apostando por el primer ciclo de Educación Infantil. CEE participación Educativa, 12 (monográfico Una mirada a la Educación Infantil), 8-19.

Pérez López, C. (2009). Técnicas de análisis de datos con SPSS 15. Madrid: Pearson Educación S.A. 
Pérez-Díaz, V., Rodríguez, J.C. \& Sánchez, L. (2001). La familia española ante la educación de sus hijos. Barcelona: Fundación 'La Caixa'.

Real Academia Española (2005). Diccionario panhispánico de dudas. Consultado en http://lema.rae.es/dpd/srv/search?id=Tr5x8M FOuD6DVTIDBg

Reparaz, C. \& Naval, C. (2014). Bases conceptuales de la participación de las familias. En Consejo Escolar del Estado (Coord.), La participación de las familias en la educación escolar (pp. 21-34). Madrid: Ministerio de Educación, Cultura y Deporte.

Rodríguez, R.M. (2010a). Éxito académico de la segunda generación de inmigrantes en EE.UU. Revista Española de Educación Comparada, 16, 329-355. doi: http://doi.org/10.5944/reec.16.2010.7535

Rodríguez, R.M. (2010b). Éxito académico de los estudiantes inmigrantes. Factores de riesgo y de protección. Educación XX1, 13(1), 101-123. doi: http://doi.org/10.5944/educxx1.13.1.279

Santín, D. (2001). Influencia de los factores socioeconómicos en el rendimiento escolar internacional: hacia la igualdad de oportunidades educativas. Documentos de trabajo de la Facultad de Ciencias Económicas y Empresariales. Madrid: Universidad Complutense.

Santos, M.A. \& Lorenzo, M. (2009). La participación de las familias inmigrantes en la escuela. Un estudio centrado en la procedencia. Revista de Educación, 350, 277300.

Santos, M.A., Esteve, J.M., Ruiz, C. \& Lorenzo, M. M. (2004). Familia, educación y flujos migratorios. En J.M. Touriñán \& M.A. Santos (Eds.), Familia, educación y sociedad civil (pp. 203-280). Universidad de Santiago de Compostela.

Sheldon, S.B. \& Epstein, J.L. (2002). Improving student behavior and school discipline with family and community involvement. Education and Urban Society, 35(1), 4-26. doi: https://doi.org/10.1177/001312402237212

\section{Notas}

1.El presente trabajo es derivación de una investigación promovida y sufragada por el Consejo Escolar de Estado.

2. Siguiendo las recomendaciones de la Real Academia Española (2005), en este artículo se ha utilizado el masculino genérico para referirse indistintamente a ambos sexos. 


\begin{tabular}{|c|c|}
\hline Autores / Authors & $\begin{array}{c}\text { To know more / } \\
\text { Saber más }\end{array}$ \\
\hline Parra, Joaquín (jparra@um.es) & ORCID \\
\hline $\begin{array}{l}\text { Doctor en Pedagogía, Profesor Titular del Departamento de Métodos de } \\
\text { Investigación y Diagnóstico en Educación (MIDE), Universidad de Murcia. Líneas } \\
\text { de investigación: relaciones familia-centro educativo y diagnóstico en educación. } \\
\text { Dirección: Universidad de Murcia. Facultad de Educación, Campus de Espinardo, } \\
30100 \text { Murcia (España) }\end{array}$ & $\underline{0000-0001-6818-8909}$ \\
\hline Gomariz, María-Ángeles magovi@um.es & ORCID \\
\hline $\begin{array}{l}\text { Doctora en Pedagogía, Profesora Contratada del Departamento de MIDE, } \\
\text { Universidad de Murcia. Líneas de investigación: relaciones familia-centro } \\
\text { educativo, orientación educativa, acción tutorial y Diagnóstico en Educación. } \\
\text { Dirección: Universidad de Murcia. Facultad de Educación, Campus de Espinardo, } \\
30100 \text { Murcia (España). }\end{array}$ & $\underbrace{}_{\text {acodemico }}$ \\
\hline Hernández-Prados, María-Ángeles mangeles@um.es & \\
\hline $\begin{array}{l}\text { Doctora en Pedagogía, Profesora Contratada del Departamento de Teoría e } \\
\text { Historia de la Educación, Universidad de Murcia. Líneas de investigación: } \\
\text { Educación familiar, educación en valores, ciudadanía, convivencia y sociedad de la } \\
\text { información. Dirección: Universidad de Murcia. Facultad de Educación, Campus de } \\
\text { Espinardo, } 30100 \text { Murcia (España). }\end{array}$ & ResearchGate \\
\hline García-Sanz, Mari-Paz maripaz@um.es & ORCID \\
\hline $\begin{array}{l}\text { Doctora en Pedagogía, Profesora Titular del Departamento de Métodos de } \\
\text { Investigación y Diagnóstico en Educación de la Universidad de Murcia. Líneas de } \\
\text { investigación: relaciones familia-centro educativo, evaluación de programas } \\
\text { educativos y planificación, aprendizaje y evaluación de competencias Dirección: } \\
\text { Universidad de Murcia. Facultad de Educación, Campus de Espinardo, } 30100 \\
\text { Murcia (España). }\end{array}$ & ResearchGate \\
\hline
\end{tabular}

\section{RELIEVE}

\section{Revista ELectrónica de Investigación y $\mathbf{E V}$ aluación Educativa E-Journal of Educational Research, Assessment and Evaluation}

[ISSN: 1134-4032]

\footnotetext{
(C) Copyright, RELIEVE. Reproduction and distribution of this articles it is authorized if the content is no modified and their origin is indicated (RELIEVE Journal, volume, number and electronic address of the document).

(C) Copyright, RELIEVE. Se autoriza la reproducción y distribución de este artículo siempre que no se modifique el contenido y se indique su origen (RELIEVE, volumen, número y dirección electrónica del documento).
} 